\title{
ANÁLISE DA REMODELAÇÃO CARDÍACA EM ANIMAIS COM INSUFICIÊNCIA CARDÍACA (IC) INDUZIDA PELA MONOCROTALINA SUBMETIDOS A TREINAMENTO FÍSICO PREVENTIVO
}

Ana Karênina Dias de Almeida Sabela ${ }^{1}$, Thaoan Bruno Mariano ${ }^{1}$, André Casanova de Oliveira ${ }^{2}$, Patrícia Mayumi Takamoto ${ }^{3}$, Mariana Fernandes Pellosi ${ }^{3}$, Nair Karina de Oliveira ${ }^{3}$, Thays da Silva Garrido $^{3}$, Luiz Carlos Marques Vanderlei ${ }^{5}$, Edna Maria do Carmo ${ }^{5}$, Francis Lopes Pacagnelli ${ }^{6}$

${ }^{1,6}$ Universidade do Oeste Paulista - UNOESTE, Programa de Pós-Graduação em Ciência Animal, ${ }^{3,6}$ Curso de Fisioterapia. Presidente Prudente - SP. ${ }^{2}$ Faculdade de Medicina. Especialista em Fisioterapia Hospitalar, São José do Rio Preto - SP.

${ }^{4}$ Universidade Estadual Paulista - FCT/UNESP. Departamento de Fisioterapia, Presidente Prudente - SP. Presidente Prudente - SP. E-mail: karenina almeida@hotmail.com.

\section{RESUMO}

A prevalência de Insuficiência Cardíaca (IC) aumenta anualmente, sendo uma das razões o aumento da expectativa de vida. A IC Direita (ICD) apresenta uma grande morbi-mortalidade, estando principalmente relacionada com Cor Pulmonale. Inexistem estudos que avaliam repercussões do treinamento físico preventivo (ICD). Objetivo deste estudo foi avaliação/impacto do treinamento físico preventivo em ratos com ICD induzida por monocrotalina. Foram utilizados ratos Wistar machos, divididos em 4 grupos de 8 animais: grupo sedentário controle (C); grupo treino controle $(\mathrm{T})$; grupo sedentário monocrotalina $(\mathrm{M})$; grupo treino monocrotalina (TM). $\mathrm{O}$ protocolo de treino foi realizado em esteira por 13 semanas, 5 vezes/semana (10 semanas de treino preventivo e 3 semanas após a injeção de monocrotalina). Após este período os animais foram mortos e foram analisados os parâmetros anatômicos do coração, pulmão e fígado. Concluise que os animais apresentaram disfunção ventricular direita e que o treinamento preventivo não trouxe benefícios neste parâmetro cardíaco.

Palavras-chave: insuficiência cardíaca; monocrotalina; ratos wistar; condicionamento físico animal; prevenção de doenças.

\section{ANALYSIS OF CARDIAC REMODELING IN ANIMALS WITH HEART FAILURE (HF) INDUCTED BY MONOCROTALINE SUBMITTED PREVENTIVE PHYSICAL TRAINING}

\begin{abstract}
The prevalence of Heart failure (HF) increases annually, being one of the reasons the raise of life expectancy. The Right Heart Failure (RHF) presents a big morbidity-mortality, being mainly related with Cor Pulmonale. There aren't studies that evaluate repercussions of preventive physical training (RHF). The goal of this study was evaluation/impact of preventive physical training in rats with RHF inducted by monocrotaline. They used males Wistar rats, divided in 4 groups of 8 animals: control sedentary $(C)$; control training $(T)$; monocrotaline sedentary $(M)$; monocrotaline training (TM). The protocol of training was realized in treadmill for 13 weeks, 5 fold/week (10 weeks of preventive training and 3 weeks after the monocrotaline shot). After this period the animals were killed and were analysed the anatomic parameters of heart, lung and liver. In conclusion the animals presented right ventricular dysfunction and the preventive training didn't bring benefits in this cardiac parameter.
\end{abstract}

Keywords: heart failure; monocrotaline; rats wistar; physical conditioning animal; disease prevention. 


\section{INTRODUÇÃO}

A prevalência estimada de Insuficiência Cardíaca (IC) no mundo é de 23 milhões de pessoas e cerca de dois milhões de novos casos são diagnosticados anualmente (BATLOUNI et al., 1999; NETO, 2004; NOGUEIRA et al., 2010). Segundo dados do DATASUS, há no Brasil cerca de dois milhões de pacientes com IC, sendo diagnosticados 240 mil de novos casos por ano (GUIMARÃES, 2002; NOGUEIRA et al., 2010). As projeções indicam que, em 2025, o Brasil terá a sexta maior população de idosos, aproximadamente, 30 milhões de pessoas (15\% da população total). Isso deve resultar na multiplicação dos casos de IC e dos gastos com essa síndrome (GUIMARÃES 2002; NOGUEIRA et al., 2010).

Os estudos relacionados a IC, a maioria descreve a alteração no ventrículo esquerdo. Entretanto, a IC caracterizada pela falência do ventrículo direito corresponde a cerca de $30 \%$ e é a principal causa de morbidade e mortalidade pós transplante cardíaco e insuficiência cardíaca congestiva (BRAUNWALD et al., 2003; RABBO 2005). Esses estudos não consideram a IC direita (ICD) causada pela hipertensão arterial pulmonar (secundárias) e cor pulmonale, que aumentaria ainda mais este índice (BRAUNWALD et al., 2003).
Um levantamento feito em hospitais da América do Norte indicou que a prevalência de hipertensão arterial pulmonar por doenças respiratórias crônicas corresponde à aproximadamente $28 \%$ e que doença pulmonar obstrutiva crônica (DPOC) é a causa mais comum de "cor pulmonale" (MINAI, 2010).

A remodelação cardíaca tem um papel importante na fisiopatologia da disfunção ventricular que ocorre em diversos modelos de agressão cardíaca (ZORNOFF et al., 2009; FARAH et al., 2013).

O exercício físico tem sido considerado uma terapêutica não farmacológica eficaz para minimizar as alterações cardíacas e disfuncionais que esta doença ocasiona. A atividade física realizada de maneira adequada por profissionais especializados contribui para ajudar nos tratamentos não farmacológicos de doenças cardiovasculares, tornando a inatividade física desnecessária e indesejável (RABBO, 2005).

O treinamento físico realizado em ratos (natação) observou que o exercício regular após o infarto do miocárdio (IM), pode atenuar os indicadores de IC, contribuindo para uma melhora no remodelamento cardíaco (PORTES; TUCCI, 2006).

Em um estudo realizado em ratas, não foi observado melhora no tamanho do 
infarto no exercício físico preventivo (natação) ao IM, mantendo a função sistólica e diastólica prejudicadas, mesmo após 1 semana que realizou-se a estenose aórtica para a induzir o IM (VEIGA et al., 2011). Da mesma forma, o exercício físico realizado preventivamente ao $\mathrm{IM}$, não diminuiu as alterações encontradas, tais como, o tamanho do infarto, congestão pulmonar, remodelamento cardíaco e disfunções sistólica e diastólica (VEIGA et al., 2013).

Entretanto, avaliar os efeitos preventivos do exercício físico na remodelação cardíaca tem sido pouco estudado e os resultados são controversos. 0 objetivo do estudo foi avaliar os efeitos do treinamento físico preventivo na remodelação cardíaca de ratos com ICD.

\section{METODOLOGIA}

\section{Animais e Procedimentos}

Foram utilizados 32 ratos Wistar, machos, adultos, com peso entre 160 e 235 gramas. Estes animais são provenientes do Biotério Central da Universidade do Oeste Paulista - UNOESTE de Presidente Prudente São Paulo. Os protocolos experimentais que foram utilizados neste estudo estão em conformidade com os princípios de cuidados com animais de laboratório formulado pelo Colégio Brasileiro de Experimentação Animal (COBEA) e pelo Comitê de Ética da Universidade do Oeste Paulista - UNOESTE.
O projeto foi cadastrado e aprovado sob número 1838 e 1839. Os animais foram mantidos em gaiolas com dimensão $41 \times 34 \times 16 \mathrm{~cm}$, onde mantiveram 3 animais por gaiola, à temperatura de 21 à 23 으 e com ciclos de luminosidade de $12 \mathrm{~h}$ (claro (escuro). Os ratos receberam ração e água ad libitum.

Os animais foram divididos de forma aleatória em 4 grupos experimentais com 8 animais da seguinte forma:

- Grupo sedentário controle (C);

- Grupo treino controle (T);

- Grupo sedentário monocrotalina (M);

- Grupo treino monocrotalina (TM).

O treinamento foi realizado em uma esteira rolante, onde realizamos 10 semanas de pré treinamento, após isso, foi injetado soro fisiológico nos animais dos grupos C e T e monocrotalina nos animais dos grupos $\mathrm{M}$ e TM.

Em seguida, foi realizado o teste de velocidade máxima para dosar o limiar do lactato e estabelecer a velocidade de treinamento dos grupos (realizado 24 após injeção da monocrotalina, depois ao final da segunda e terceira semana) e posteriormente após a injeção da droga, foram realizados mais 3 semanas de treinamento aeróbio.

Passados 15 dias, os animais foram mortos e seus órgãos (coração, sendo átrios, ventrículo direito (VD), ventrículo esquerdo (VE), pulmão e fígado) foram retirados, 
pesados para analisarmos os dados anatômicos.

\section{Treinamento}

Todos os animais foram colocados para andar na esteira rolante adaptada para roedores (confeccionada no SEBRAE de Presidente Prudente, SP). Foi feito uma seleção dos ratos que tiveram melhor desempenho em realizar o treinamento.

Os animais dos grupos T e TM foram submetidos a um treinamento aeróbio em esteira rolante, sendo 2 semanas de pré treinamento, onde o tempo e a velocidade foi aumentado gradativamente, com início do treino à $0,6 \mathrm{~km} / \mathrm{h}$ em 15 minutos no primeiro dia e terminando a segunda semana de pré treinamento com velocidade de $0,9 \mathrm{~km} / \mathrm{h}$ em 45 minutos (RODRIGUES et al., 2007).

Após o final do período de pré treinamento, iniciou-se o período de treinamento, que constituiu 8 semanas de treinamento aeróbio. Este protocolo foi adaptado de acordo com a máxima fase estável do lactato (MACHADO et al., 2006), de forma progressiva, para animais com ICD causada pela monocrotalina. A primeira semana de treinamento aeróbio foi realizada 45 minutos à $0,9 \mathrm{~km} / \mathrm{h}$, sendo os 5 minutos iniciais (aquecimento) e finais (desaceleração) com velocidade de 0,6 km/h. A segunda e terceira semanas o treinamento foi realizado com velocidade de $0,9 \mathrm{~km} / \mathrm{h}$ por
60 minutos. A quarta, quinta e sexta semanas o treinamento foi realizado com velocidade de $1,0 \mathrm{~km} / \mathrm{h}$ em 60 minutos. A sétima e oitava semanas o treinamento foi realizado com velocidade de $1,1 \mathrm{~km} / \mathrm{h}$ em 60 minutos.

\section{Desenho do estudo}

Indução da IC

Nos animais do grupo $C$ e $T$ foi realizado administração de solução salina, para que todos os animais fossem avaliados com o mesmo grau de estresse devido administração de injeção. A IC foi induzida nos animais experimentais nos grupos $\mathrm{M}$ e TM. Foi injetada uma única dose intraperitoneal da monocrotalina na proporção 60mg/kg (COLOMBO et al., 2013).

\section{Controle de Ração}

Após a indução da IC, durante as 3 semanas de treinamento aeróbio os animais foram separados em gaiolas individuais para dosar o consumo de ração diário. Os Grupos M e TM receberam comida a vontade, já que estes animais vão diminuindo o consumo de ração devido a IC. Nos Grupos C e T, os animais receberam a média de consumo dos animais dos outros 2 Grupos.

\section{Limiar do Lactato}

Vinte e quatro horas após a injeção da monocrotalina foi realizado o Teste de Velocidade Máxima para analisar o Limiar do 
Lactato (LL) e verificar a velocidade de treinamento que os animais deveriam realizar o treinamento.

Foi realizada a dosagem do $\mathrm{LL}$ e calculado a média da velocidade em que os animais deveriam andar nas próximas 3 semanas de treino aeróbio. Para a realização do Teste de Velocidade Máxima foi feito equiparação dos parâmetros onde convertemos $\mathrm{m} / \mathrm{seg}$ para $\mathrm{km} / \mathrm{h}$. No teste de velocidade máxima a velocidade da esteira iniciou com 2 minutos de aquecimento a 0,5 $\mathrm{km} / \mathrm{h}$, seguido de 5 minutos de descanso. Logo após iniciou-se o Teste com velocidade inicial de $0,7 \mathrm{~km} / \mathrm{h}$ por 3 minutos. A velocidade foi aumentada de $0,2 \mathrm{~km} / \mathrm{h}$ gradativamente a cada 3 minutos até atingir o valor de1 $\mathrm{mM}$, comparado com o valor inicial do lactato ou até a exaustão (BECH et al., 1990; SVEDAH; MACINTOSH, 2003). lactato foi mensurado ao final de cada ciclo de 3 minutos pela coleta de sangue da cauda do rato utilizando o aparelho Lactimetro Accutrend Plus $\quad\left(\right.$ Roche $^{\circledR}$ ) devidamente calibrado. O cálculo para estipular a velocidade máxima foi realizado com uma média aritmética de todas as velocidades de cada grupo experimental ao atingirem o LL. Este teste foi repetido ao final da 12a. e 13a. semanas para ajustar a carga de treino.

O Grupo $T$ realizou o treinamento aeróbio na 11a. e 12a. semanas com velocidade média de $0,9 \mathrm{~km} / \mathrm{h}$. O LL foi 2,5mM. O Grupo TM treinou com velocidade $0,8 \mathrm{~km} / \mathrm{h}$ e o LL foi $3,3 \mathrm{mM}$.

O Grupo $\mathrm{T}$ realizou o treinamento aeróbio na 13ạ semana com velocidade média $0,9 \mathrm{~km} / \mathrm{h}$ e o LL foi $2,8 \mathrm{mM}$. O Grupo TM andou com velocidade de $0,9 \mathrm{~km} / \mathrm{h}$ e o LL foi $2,6 \mathrm{mM}$.

O Grupo T teve LL média de 2,5 mM e o Grupo TM o LL foi 3,3 mM.

\section{Avaliação da Remodelação Cardíaca}

Os animais foram mortos, o coração foi retirado, dissecado, separando átrios, VD e VE (COLOMBO et al., 2013). Os parâmetros anatômicos, peso úmido do VD e do VE foi normalizado pelo peso corpóreo final do rato (PC), que foi utilizado como índice de hipertrofia ventricular. A insuficiência cardíaca foi avaliada pela retenção hídrica por meio da relação peso úmido/peso seco do pulmão e fígado (CARVALHO et al., 2010).

\section{Análise estatística}

Para análise da normalidade foi utilizado o teste de Shapiro Wilk. Os dados foram expressos em média \pm desvio padrão, mediana (mínimo-máximo). Para comparação entre os grupos foram utilizados o teste de Kruskal-Wallis seguido de pós teste de Dunn's ou ANOVA (One way). Foi considerado significante o valor de $p<0,05$ 


\section{RESULTADOS}

A tabela 1 mostra os dados anatômicos dos grupos C, M, T e TM. Após 35 dias, os animais que receberam a monocrotalina apresentaram sinais de disfunção cardíaca no exame pós morte, tais como: hipertrofia ventricular direita, ascite, derrame pleural e congestão hepática. Os animais dos grupos controles não apresentaram alterações.

Não houve diferença significativa nos índices do VE/PCT, Pulmão úmido/seco e Fígado úmido/seco.

Foi observada diferença estatística nos índices do Átrio/PCF e VD/PCF entre os grupos $M$ vs. C; $M$ vs. T e TM vs. C.

Tabela 1. Dados anatômicos expressos em média, \pm desvio padrão, mediana, mínimo e máximo.

VARIÁVEIS

\section{Grupos}

\begin{tabular}{ccccc} 
& $\mathbf{C}$ & $\mathbf{M}$ & $\mathbf{T}$ & $\mathbf{T M}$ \\
\hline & $0,20 \pm 0,03$ & $0,35 \pm 0,16^{*}$ & $0,20 \pm 0,06$ & $0,27 \pm 0,06 \#$ \\
ÁTRIO/PCT (g) & 0,2 & 0,31 & 0,21 & 0,25 \\
& {$[0,18-0,28]$} & {$[0,20-0,70]$} & {$[0,09-0,28]$} & {$[0,20-0,43]$} \\
VD/PCT (g) & $0,44 \pm 0,05$ & $0,81 \pm 0,30 *$ & $0,46 \pm 0,04$ & $0,75 \pm 0,21 \#$ \\
& 0,43 & 0,78 & 0,46 & 0,74 \\
VE/PCT (g) & {$[0,37-0,53]$} & {$[0,47-1,18]$} & {$[0,39-0,54]$} & {$[0,51-1,15]$} \\
& $1,85 \pm 0,07$ & $1,87 \pm 0,11$ & $1,85 \pm 0,10$ & $1,75 \pm 0,15$ \\
& 1,85 & 1,86 & 1,85 & 1,76 \\
& {$[1,73-1,95]$} & {$[1,72-2,00]$} & {$[1,65-2,05]$} & {$[1,45-1,93]$} \\
& & & & \\
Pulmão úmido/seco (g) & $4,45 \pm 0,72$ & $4,84 \pm 0,32$ & $4,72 \pm 0,33$ & $4,04 \pm 1,07$ \\
& 4,81 & 4,90 & 4,87 & 4,57 \\
& {$[3,22-5,05]$} & {$[4,17-5,12]$} & {$[4,07-5,08]$} & {$[2,65-5,26]$} \\
& & & & \\
& $4,37 \pm 2,99$ & $3,36 \pm 0,18$ & $03,29 \pm 0,29$ & $3,32 \pm 0,04$
\end{tabular}

C: Sedentário Controle; M: Sedentário Monocrotalina; T: Treino Controle; TM: Treino Monocrotalina; PCT: peso corporal total; VD: ventrículo direito; VE: ventrículo esquerdo; g: gramas. * M vs. C; M vs. T; \# TM vs. C.

\section{DISCUSSÃO}

Neste estudo constatou-se que o treinamento aeróbio realizado de forma preventiva à disfunção ventricular direita induzida por hipertensão pulmonar não produziu um efeito cardioprotetor, não reverteu a hipertrofia de átrios e VD, quando comparado com animais sedentários. Embora estudos comprovem que o exercício aeróbio seja uma forma não medicamentosa e eficaz 
para proteger o coração frente a uma agressão melhorando o remodelamento cardíaco (PORTES ; TUCCI, 2006), há pesquisas que também não evidenciaram benefícios no remodelamento em animais que realizaram treinamento preventivo no Infarto do Miocárdio (VEIGA et al., 2011; VEIGA et al., 2013).

Em outro estudo constatou que os animais espontaneamente hipertensos que realizaram treinamento aeróbio por 8 semanas tiveram benefícios no remodelamento cardíaco e na função contrátil no miócito do VE. Após 4 semanas sem realizar atividade física aeróbia, promoveu reversibilidade destes benefícios (CARNEIRO-JÚNIOR et al., 2010). Como os animais dos grupos T e TM permaneceram 2 semanas sem realizarem o treinamento aeróbio (períodos este que finalizamos o protocolo de 3 semanas de treino após a aplicação da monocrotalina e até os animais apresentarem sinais clínicos de IC e realizarmos a eutanásia), este período pode ter sido influenciado pelo mecanismo de reversibilidade cardíaca, favorecendo assim a perda dos mecanismos cardioprotetores que o treinamento aeróbio poderia ter feito ao coração.

Ao analisar os pesos do VE/PCT, Pulmão Úmido/Seco e Fígado Úmido/Seco não apresentaram diferença significativa, consequentemente, os animais não mostraram sinais evidentes de IC, como derrame pleural e congestão hepática. Porém, ao avaliar os dados dos Átrios/PCF e VD/PCF constatamos que os animais apresentaram uma disfunção cardíaca, pois seus parâmetros foram compatíveis com o de uma ICD, como sinais observados nos aumentos dos átrios e aumento do VD.

Estudos que usaram a monocrotalina para causar a ICD utilizaram animais jovens de 140 gramas ( $\pm 15 \mathrm{~g}$ ) e uma única dose de $60 \mathrm{mg} / \mathrm{kg}$ e após 3 semanas depois da aplicação da monocrotalina os animais foram mortos e a ICD foi constatada (COLOMBO et al., 2013). No nosso estudo os animais pesavam em média 347 gramas no grupo $\mathrm{M} \mathrm{e}$ 359 gramas no grupo TM e já eram adultos. A quantidade da monocrotalina utilizada nos animais foi a mesma e mensurada de forma proporcional ao peso corporal final, do dia da aplicação da droga. Porém, acreditamos que pelo fato dos nossos animais estarem com o peso bem acima no momento da injeção da monocrotalina, a droga demorou mais tempo para fazer efeito, e, além do peso, a idade pode ter influenciado, visto que os nossos animais eram mais velhos, sendo que as diferenças metabólicas agirem diretamente no metabolismo destes ratos.

Mais estudos são necessários com diferentes tipos de exercícios preventivos e por períodos mais prolongados para avaliar a resposta do remodelamento cardíaco 
patológico na disfunção cardíaca ocasionada por hipertensão pulmonar.

\section{CONCLUSÃO}

O exercício físico realizado de forma preventiva à ICD não trouxe melhora significativa nos mecanismos de proteção cardíacos relacionados a hipertrofia atrial e ventricular direita.

\section{REFERÊNCIAS}

BATLOUNI, M. et al. Sociedade Brasileira de Cardiologia. II Diretrizes da Sociedade Brasileira de Cardiologia para o diagnóstico e tratamento da insuficiência cardíaca. História natural da remodelação miocárdica: da agressão aos sintomas. Arq. Bras. Cardiol., v.72, (supl. 1), p.4-30, 1999.

BECH, O.M.; SORENSEN, J.D.; JENSEN, M.K.; DIAMANT, B.; STEINESS, E. Effects of longterm coenzyme Q10 and captopril treatment on survival and functional capacity in rats with experimentally induced heart infarction. J. Pharm. Exp. Therap., p.255-346, 1990.

BRAUNWALD, E.; ZIPES, D.P.; LIBBY, P. Tratado de medicina cardiovascular. 6. ed. São Paulo: Roca, 2003.

CARNEIRO-JÚNIOR, M.A.; PELUZIO, M.C.G.; SILVA, C.H.O.; AMORIM, P.R.S.; SILVA, K.A.; SOUZA, M.O.; ROMAN-CAMPOS, D.; PRÍMOLA-GOMES, T.N.; NATALI, A.J. Exercise Training and detraining modify the morphological and mechanical properties of single cardiac myocites obtained from spontaneously hypertensive rats. Braz J Med Biol Res., v.43, n11, p.1042-1046, 2010. DOI: 10.1590/S0100-879X2010007500117.

CARVALHO, R.F.; CASTAN, E.P.; COELHO, C.A.; LOPES, F.S.; ALMEIDA, F.L.; MICHELIN, A.; DE SOUZA, R.W.; ARAÚJO JR,
J.P.; CICOGNA, A.C.; DAL PAI, M. Heart failure increases atrogin-1 and MuRF1 gene expression in skeletal muscle with fiber typespecific atrophy. J Mol Histol., v.41, n.1, p.817, 2010. DOI: 10.1007/s10735-010-9262-x.

COLOMBO, R.; SIQUEIRA, R.; BECKER, C.U.; FERNANDES, T.G.; PIRES, C.M.; VALENÇA, S.S.; SOUZA-RABBO, M.P.; ARAÚJO, A.S.; BELLÓ-KLEIN, A. Effects of exercise on monocrotaline-induced changes in righ theart function and pulmonar artery remodeling in rats. Phisiologie e Pharmacologie, n.91, p.38-44, 2013. DOI: 10.1139/cjpp-2012-0261.

FARAH, E.; FUSCO, D.R.; OKUMOTO, P.R.R.; MINICUCCI, M.F.; AZEVEDO, O.S.; MATSUBARA, B.B.; OKOSHI, K.; ZANATI, S.G.; PAIVA, S.A.R.; ZORNOFF, L.A.M. Impact of ventricular geometric pattern on cardiac remodeling after myocardial infarction. Arq. Bras. Cardiol., v.100, n.6, p.518-23, 2013. DOI: $10.5935 / a b c .20130104$

GUIMARÃES, J.I. Sociedade Brasileira de Cardiologia. Revisão das II Diretrizes da Sociedade Brasileira de Cardiologia para o diagnóstico e tratamento da insuficiência cardíaca. Arq. Bras. Cardiol., v.79, (supl. 4), p.1-30, 2002.

MACHADO, F.B.; GOBATTO, C.A.; CONTARTESE, R.V.L.; PAPOTI, M.; MELLO, M.A.R. Máxima fase de estabilidad de lactato es ergómetro-dependiente en modelo experimental utilizando ratones. Rev Bras Med Esporte., v.12, n.5, p.259-262, 2006. DOI:10.1590/S1517-86922006000500007

MINAI, O. A. Hipertensão pulmonar na DPOC: revisão da literatura. PVRI REVIEW (PORTUGUESE), v.2, n.1, jan-jun., 2010.

NETO, J.M.R. A dimensão do problema da insuficiência cardíaca do Brasil e do mundo. Rev. SOCESP., v.14,n.1, p.1-7, 2004.

NOGUEIRA, P.R.; SALVADOR, R.; CORRÊA, K.S. Epidemiological, clinical e therapeutic profile 
of heart failure in a tertiary hospital. Arq. Bras. Cardiol., v.95, n.3, p.392-398, 2010.

PORTES, L.A.; TUCCI, P.J.F. Swim training attenuates myocardial remodeling and the pulmonary congestion in wistar rats with secondary heart failure to myocardial infarction. Arq. Bras. de Cardiol., v.87, n.1, p.54-59, 2006.

RABBO, M.P.S. Efeito do treinamento físico no perfil hemodinâmico e no stress oxidativo em modelo experimental de cor pulmonale. 2005. Tese (Doutorado) - Universidade Federal do Rio Grande do Sul - RS.

RODRIGUES, B.; FIGUEROA, D.M.; MOSTARDA, C.T.; HEEREN, M.Y.; IRIGOYEN, M.C.; ANGELIS, K. Maximal exercise test is a useful method for physical capacity and oxygen consumption determination in streptozotocin-diabetic rats. Cardiovasc Diabetol., v.13, n.6, p.1-7, 2007. DOI: 10.1186/1475-2840-6-38.

SVEDAH, K.; MACINTOSH, B.R. Anaerobic threshold: the concept and methods of measurement. Canad. J. Appl. Physiol., n.28, p.299-323, 2003.

VEIGA, E.C.A.; ANTONIO, E.L.; BOCALINI, D.S.; MURAD, N.; ABREU, L.C.; TUCCI, P.I.F.; SATO, M.A. Prior exercise training does not prevent acute cardiac alterations after myocardial infarction in female rats. Clinics., v.66, n.5, p.889-893, 2011. DOI: 10.1590/S180759322011000500028

VEIGA, E.C.A.; PORTES, L.A.; BOCALINI, D.S.; ANTONIO, E.L.; SANTOS, A.A.; SANTOS, M.H.; SILVA, F.A.; TUCCI, P.J.F. Cardiac implications after myocardial infarction in rats previously undergoing physical exercise. Arq. Bras. Cardiol., v.100, n.1, p.37-43, 2013.

ZORNOFF, L.A.; PAIVA, S.A.; DUARTE, D.R.; SPADARO, J. Ventricular remodeling after myocardial infarction: concepts and clinical implications. Arq. Bras. Cardiol., v.92, n.2, p.150-64, 2009. 